\title{
Co-regulated expression of HAND2 and DEIN by a bidirectional promoter with asymmetrical activity in neuroblastoma Harald Voth ${ }^{1,2}$, André Oberthuer ${ }^{1}$, Thorsten Simon ${ }^{1}$, Yvonne Kahlert ${ }^{1}$, Frank Berthold ${ }^{1}$ and Matthias Fischer*1
}

\author{
Address: ${ }^{1}$ Department of Pediatric Oncology and Hematology and Center of Molecular Medicine Cologne (CMMC), University Children's \\ Hospital, Cologne, Germany and 2Department of Dermatology, University of Bonn, Bonn, Germany \\ Email: Harald Voth - hvoth@gmx.de; André Oberthuer - andre.oberthuer@uk-koeln.de; Thorsten Simon - thorsten.simon@uk-koeln.de; \\ Yvonne Kahlert - yvonne.kahlert@uk-koeln.de; Frank Berthold - frank.berthold@uk-koeln.de; Matthias Fischer* - matthias.fischer@uk-koeln.de \\ * Corresponding author
}

Published: 6 April 2009

BMC Molecular Biology 2009, 10:28 doi:10.1 186/1471-2199-10-28

This article is available from: http://www.biomedcentral.com//47/-2/99//0/28

(c) 2009 Voth et al; licensee BioMed Central Ltd.

This is an Open Access article distributed under the terms of the Creative Commons Attribution License (http://creativecommons.org/licenses/by/2.0), which permits unrestricted use, distribution, and reproduction in any medium, provided the original work is properly cited.
Received: 12 November 2008

Accepted: 6 April 2009

\begin{abstract}
Background: HAND2, a key regulator for the development of the sympathetic nervous system, is located on chromosome 4q33 in a head-to-head orientation with DEIN, a recently identified novel gene with stage specific expression in primary neuroblastoma (NB). Both genes are expressed in primary NB as well as most NB cell lines and are separated by a genomic sequence of 228 bp. The similar expression profile of both genes suggests a common transcriptional regulation mediated by a bidirectional promoter.
\end{abstract}

Results: Northern Blot analysis of DEIN and HAND2 in 20 primary NBs indicated concurrent expression levels of the two genes, which was confirmed by microarray analysis of 236 primary NBs (Pearson's correlation coefficient $r=0.65$ ). While DEIN expression in the latter cohort was associated with stage 4S ( $p=0.02)$, HAND2 expression was not associated with tumor stage. In contrast, both HAND2 and DEIN transcript levels were highly associated with age at diagnosis $<12$ months $(p=0.001)$. The intergenic region shows substantial homology in different species $(89 \%$, $72 \%$ and $53 \%$ identity between human and mouse, chicken and zebrafish, respectively) and contains many highly conserved putative transcription factor binding sites. Using luciferase reporter gene constructs, asymmetrical bidirectional promoter activity was found in four NB cell lines: In DEIN orientation, an average 3.4 fold increase in activity was observed as compared to the promoterless vector, whereas an average 15.4 fold activation was detected in HAND2 orientation. The presence of two highly conserved putative regulatory elements, one of which was shown to enhance HAND2 expression in branchial arches previously, displayed weak repressor activity for both genes.

Conclusion: HAND2 and DEIN represent a gene pair that is tightly linked by a bidirectional promoter in an evolutionary highly conserved manner. Expression of both genes in NB is coregulated by asymmetrical activity of this promoter and modulated by the activity of two cisregulatory elements acting as weak repressors. The concurrent quantitative and tissue specific expression of HAND2 and DEIN suggests a functional link between both genes. 


\section{Background}

HAND2 is a basic helix-loop-helix transcription factor that is expressed in the developing heart, neural crest derivates and autonomic nervous system including sympathetic neurons, adrenal chromaffin cells and enteric neurons [1]. HAND2 plays a key role in the development and differentiation of the sympathetic nervous system by promoting differentiation of neural crest derived precursors into catecholaminergic neurons and maintaining the neural precursor pool of cells $[2,3]$. Functional analyses showed that HAND2 belongs to the group of BMP induced transcription factors that acts downstream of PHOX2B and upstream of GATA2, GATA3, PHOX2A and the noradrenergic marker genes Tyrosine Hydroxylase (TH) and Dopamine Beta Hydroxylase (DBH) [3,4]. In addition, HAND2 is highly expressed in neuroblastoma (NB), a pediatric tumor arising from undifferentiated migrating sympathetic precursor cells that shows conspicuously divergent biological phenotypes ranging from spontaneous regression to rapid progression and metastasis [5].

Recently, we have reported on the identification of DEIN, a novel gene with high expression in NB of children $<1$ year of age and stage $4 \mathrm{~S} \mathrm{NB}$, both of which are associated with a good prognosis and spontaneous regression [6]. Given the lack of a putative coding region, DEIN was predicted to represent a noncoding RNA whose function in NB and sympathetic nervous system development is yet unclear. DEIN is located on chromosome 4q33-34 in a head-to-head orientation with HAND2 with both genes being separated by a nucleotide sequence of $228 \mathrm{bp}$. The close vicinity and divergent configuration of both genes together with their concurrent tissue specific expression pattern suggests a common transcriptional regulation that is mediated by the intergenic sequence. Bidirectional gene organisation was shown to represent a common feature in the human genome with approximately $10 \%$ of all genes showing this configuration. Many of these gene pairs were shown to be co-regulated and/or functionally related [7].

This study aimed to examine the potential of the intergenic region to direct transcription in HAND2 and DEIN orientation, thereby acting as a bidirectional promoter. In addition, conservation and structural features such as putative transcription factor binding sites (TFBS) of the genomic region were analyzed. To test whether the expression of both genes is co-regulated in NB in vivo and associated with prognostic factors, expression levels of DEIN and HAND2 were examined in a cohort of 236 primary NBs of different stages by microarray analysis.

\section{Results \\ Determination of transcriptional start sites and the nucleotide structures of HAND2 and DEIN transcripts in NB} The human HAND2 gene was initially cloned from a human fetal cardiac cDNA library by Russel et al. [8]. The
$1.3 \mathrm{~kb}$ long consensus sequence derived from cDNA clones $\underline{\mathrm{AF} 087940}$ and $\mathrm{AF087941}$ was shorter than the 2.3 $\mathrm{kb}$ transcript detected in Northern Blot, and the authors expected the unaccounted sequence to be located in the 3 '-UTR of the gene. However, numerous transcribed sequences containing the coding region of HAND2 and extending several $100 \mathrm{bp}$ in 5 '-direction have been submitted to GenBank in recent years suggesting that the unaccounted sequence is most probably located upstream of the coding region. In order to determine the transcriptional start site and the full length sequence of the main HAND2 transcript in NB, we performed a combined RACE- and RT-PCR approach. The resulting transcript is $2351 \mathrm{bp}$ long and comprises two exons of 1495 and 856 bp (GenBank accession FJ226608). The 5'-sequence of the first exon extends into a CpG island with an average GCcontent of $70 \%$ which might have impeded the full-length cloning of the human transcript in previous attempts. Our results are well in line with the reported length of $2.3 \mathrm{~kb}$ by Northern Blot hybridization from heart tissue [8] and our Northern Blot analyses. The genomic structure of HAND2 with a long first and a shorter second exon with the coding region being interrupted by an intron is highly conserved in other species such as mouse, rat, chicken and zebrafish (data not shown). Furthermore, the transcriptional start site of HAND2 is highly conserved in all of these species. The 5'-end of FJ226608 is identical with the start sites of mRNA AK122739 and EST DA609347, both of which were derived from NB cell line IMR-32. In contrast, the HAND2 reference sequence NM 021973, which has not been experimentally confirmed, lacks two nucleotides at its $5^{\prime}$-end as compared to our transcript. The cloning procedures and the nucleotide structures of the DEIN transcript variants have been described in detail previously [6].

\section{Quantitative expression of HAND2 and DEIN in primary NB}

To determine whether expression of HAND2 and DEIN is co-regulated in neuroblastoma, we performed Northern Blot analysis of 20 primary NB with HAND2- and DEINspecific cDNA probes. We detected strong expression of both genes in all stage $4 \mathrm{~S}$ tumors, moderate to low expression in localized tumors, and little or no expression in stage 4 tumors (fig. 1). In NB cell lines, expression of DEIN and HAND2 was detected in cell lines SH-SY5Y and Kelly, but it was absent in cell line SH-EP. The concurrent transcript levels of HAND2 and DEIN in primary NB and NB cell lines indicated a co-regulated expression of both genes in NB.

To validate these results, expression of DEIN and HAND2 was analyzed in a set of 236 primary NB samples by microarray analysis (see Additional file 1). Patients diagnosed before the age of one year had significantly higher DEIN and HAND2 expression values as compared to 


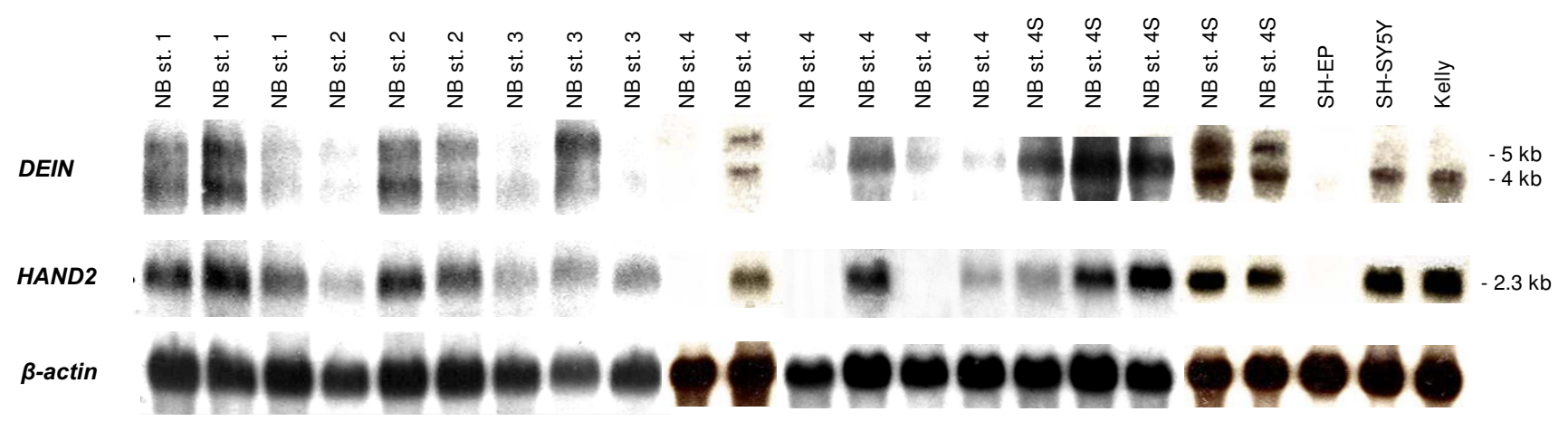

Figure I

Northern Blot analysis of HAND2 and DEIN expression in primary NB and NB cell lines. Ten $\mu g$ of total RNA from 20 primary NBs (stage I, $n=3$; stage $2, n=3$; stage $3, n=3$; stage $4 S, n=5$; stage $4, n=6$ ) and NB cell lines SH-EP, SH-SY5Y and Kelly were blotted and hybridized with HAND2- and DEIN-specific cDNA-probes; $\beta$-actin was used as loading control.

A)

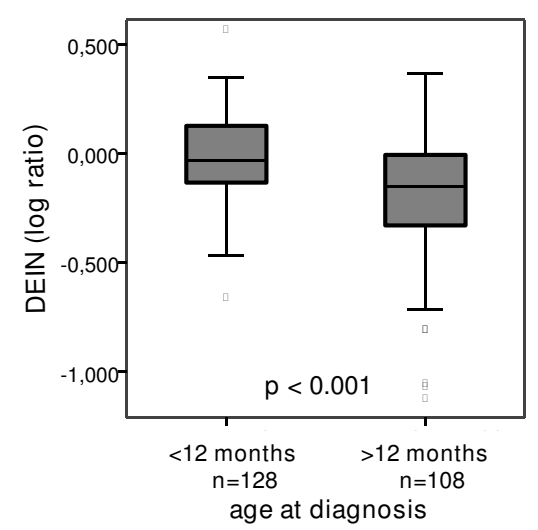

B)

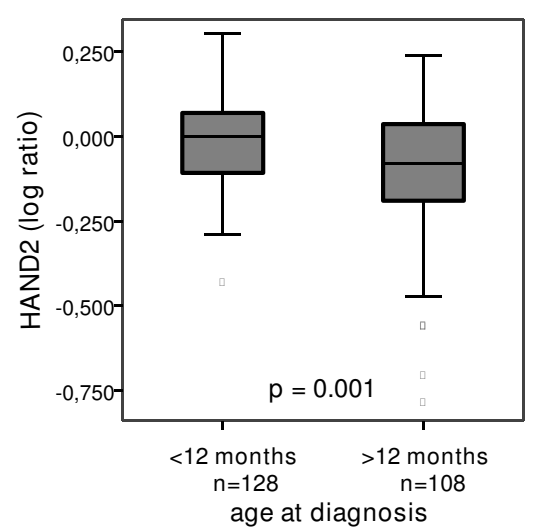

C)

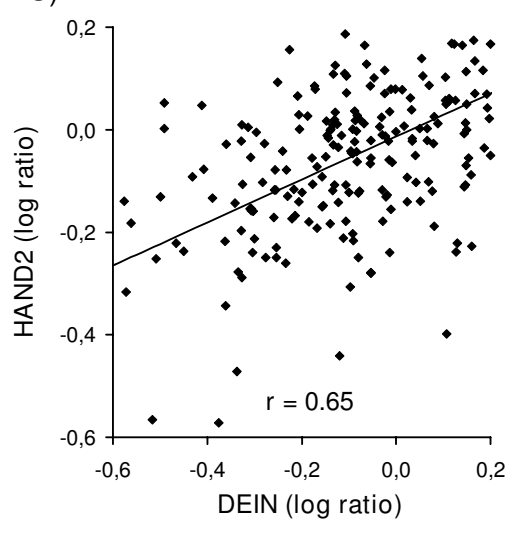

Figure 2

DEIN and HAND2 expression in 236 primary neuroblastomas as determined by microarray analysis. Relative expression levels of DEIN (A) and HAND2 (B) in neuroblastoma subsets according to age at diagnosis. Scatter Plot showing correlation of relative expression values for HAND2 and DEIN (C); $r$, Pearson's correlation coefficient.

patients diagnosed later (both $\mathrm{p}=0.001$; fig. $2 \mathrm{~A}$ and $2 \mathrm{~B}$ ). Expression of both genes was also significantly higher in children diagnosed before the age of 18 months as compared to older patients (both $\mathrm{p}=0.01$; data not shown). As expected, DEIN expression was significantly higher in tumors of stage $4 \mathrm{~S}$ than in those of stage $4(\mathrm{p}=0.02)$. These results are in line with our previous study that reported on a strong association between DEIN expression and stage $4 \mathrm{~S}$ as well as age $<1$ year in a cohort of 121 primary NBs by real-time RT-PCR [6]. However, in contrast to the results of our previous study [6], DEIN expression was neither significantly different between stage 4 and localized stages, nor between stage $4 \mathrm{~S}$ and localized stages in the micoarray analyses (data not shown). This discrepancy might be explained by the high percentage of patients $<12$ months of age among localized tumors in the microarray cohort (81/119 patients, 68\%). HAND2 mRNA levels were not associated with either stage, which is in agreement with a study of Gestbloom et al., who reported that HAND2 is expressed independently of tumor stage or differentiation in NB [5]. Nevertheless, HAND2 and DEIN expression values were well correlated in our study (Pearson's correlation coefficient $r=0.65$, fig. $2 \mathrm{C}$ ). Together, these findings support the hypothesis that expression of HAND2 and DEIN is co-regulated in primary NB.

\section{Characterization of the putative bidirectional promoter sequence between HAND2 and DEIN}

The putative promoter between HAND2 and DEIN is located within a CpG island (GC-content of 70\%) that extends into the first exon of HAND2 (fig. 3). The inter- 
genic region shows high evolutionary conservation with 89, 71 and $53 \%$ nucleotide identity of the human sequence in comparison to mouse, chicken and zebrafish, respectively. The high degree of homology is comparable to that of the coding region of HAND2 which shows 92, 88 and $68 \%$ identity of the human sequence in comparison to mouse, chicken and zebrafish, respectively.

No TATA-Box, but various other highly conserved potential transcription regulatory elements were found in the bidirectional promoter (fig. 4). These include two GCboxes with SP-1 sites and one AP- 1 site in DEIN-orientation as well as one AP-2 site in HAND2-orientation. Four binding sites for zinc finger transcription factors GATA-1 or -2 (two each in DEIN and HAND2 orientation, respectively) were detected. We also found four binding sites for homeobox transcription factors including three binding sites for CdxA (one in HAND2 orientation and two in DEIN orientation), and one binding site each for Abd-B and Prx2 in HAND2 orientation. Finally, a highly conserved site for CAMP response element-binding protein (CREB) in both orientations and a highly conserved centrally located CCAAT box in HAND2 orientation were detected. Evolutionary conservation was found for the CDxA, CREB and the CCAAT binding sites in all vertebrate species investigated, while SP1, AP1 and 2, Abd-B and GATA binding sites were only conserved in mouse and/or chicken.

\section{Asymmetrical activity of the bidirectional promoter between DEIN and HAND2}

To test the ability of the putative bidirectional promoter to activate transcription in DEIN and HAND2 orientation, it was cloned separately or together with two other highly conserved putative regulatory elements in both orientations into a luciferase reporter vector. A total of six reporter gene constructs were transfected into four neuroblastoma cell lines (summarized in fig. 3). It was demonstrated that the bidirectional promoter is able to drive expression in both HAND2 and DEIN direction in all cell lines with $\sim 4$ fold higher activity in HAND2 orientation than in DEIN orientation. In DEIN orientation the promoter caused a $2.2-5$ fold activation (mean value, 3.4) as compared to the promoterless vector $(\mathrm{p}<0.01$ for all cell lines except SH-SY5Y, which is probably due to the lower transfection efficiency of SH-SY5Y). A $4.9-25.5$ fold increase in activity (mean value, 15.4) was detected for the promoter in HAND2 orientation in all cell lines as compared to the promoterless vector ( $\mathrm{p}<0.01$ for all cell lines).

To test whether promoter activity is influenced by either of two other highly conserved sequence fragments that are located within the genomic sequence covered by DEIN, we cloned these elements either upstream or downstream of the luciferase gene in the respective orientations relative to the bidirectional promoter according to their actual genomic position (constructs $\mathrm{B}, \mathrm{C}, \mathrm{E}$ and $\mathrm{F}$, fig. $3 \mathrm{~A}$ ). The presence of a $90 \mathrm{bp}$ long regulatory element that was shown to act as enhancer for HAND2 expression in branchial arches [9] decreased luciferase activity in both DEIN and HAND2 orientations. For construct C, an average 2.6 fold reduction in activity was detected as compared to construct A ( $\mathrm{p}=0.05$ for SK-N-AS, SH-EP and Kelly; not significant [n.s.] for SH-SY5Y). For construct F, an average 4.4 fold reduction in activity was detected as compared to construct D ( $\mathrm{p}=0.05$ for Kelly; n.s. for $\mathrm{SH}$ SY5Y, SH-EP and SK-N-AS).

Another highly conserved sequence element of $302 \mathrm{bp}$ located in exon 5 of DEIN, which has been suggested to represent a cis-regulatory element for DEIN and/or HAND2 expression [6], also exerted weak repressor activity on both promoters. For construct B, we detected an average 1.1 fold reduction in activity as compared to construct A ( $p<0.05$ for SH-EP, SK-N-AS and Kelly; n.s. for SH-SY5Y). For construct E, we detected an average 3.2 fold reduction as compared to construct D ( $p<0.05$ for SH-EP; n.s. for SH-SY5Y, SK-N-AS and Kelly).

\section{Discussion}

In this study, we report on the molecular characterization of a highly conserved intergenic nucleotide sequence between HAND2 and DEIN which functions as a bidirectional promoter. Bidirectional gene configuration, defined as two divergently transcribed genes that are separated by less than $1 \mathrm{~kb}$, is a common architectural feature in the human genome and was shown to account for coregulated and/or tissue specific expression of functionally related genes [10]. Most of these genes have been reported to belong to the group of housekeeping genes many of which are involved in DNA repair and replication [7]. In recent years, other non-homologous bidirectional gene pairs were identified with functions in senescence [11], antigen presentation [12], brain disease $[13,14]$, oncogenesis or tumor supression [15-17].

\section{Structure and conservation of the bidirectional promoter}

The majority of bidirectional promoters are less than 300 bp in length, have a median GC-content of $66 \%$ and associate with CpG-islands in 75\% [7]. All these features are present in the common promoter region of HAND2 and DEIN, which is 228 bp long, contains 13 CpG sites and has a GC-Content of $70 \%$. Interestingly, the distance between HAND2 and DEIN remained stable throughout evolution with only 14 and 7 additional nucleotides in the human sequence as compared to chicken and mouse, respectively. Although there is no systematic evaluation on the alteration of the genomic distance between bidirectional gene pairs throughout evolution, we found examples reporting a far more dramatic increase of several hundreds of bp [11]. 
A)

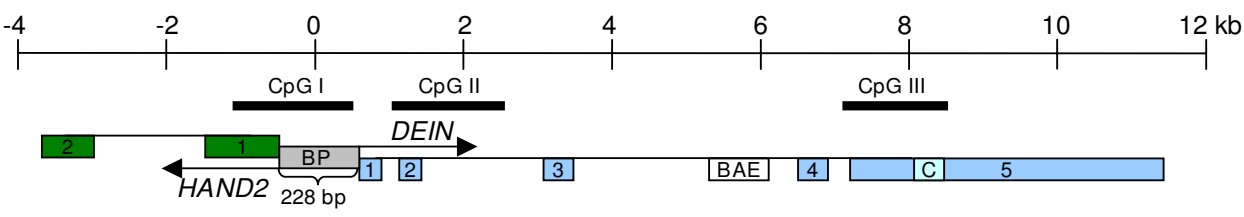

A

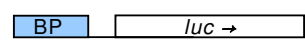

B

\begin{tabular}{|l|l|}
\hline $\mathrm{BP}$ & $\mathrm{IuC} \rightarrow$ \\
\hline
\end{tabular}

C

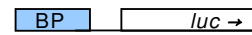

BAE

D

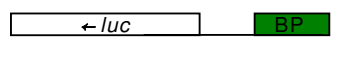

E

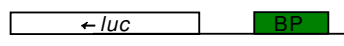

$\mathrm{F}$

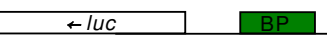

G SVE $\quad$ SluC SVP

$\mathrm{H}$

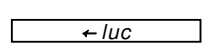

B)

SK-N-AS

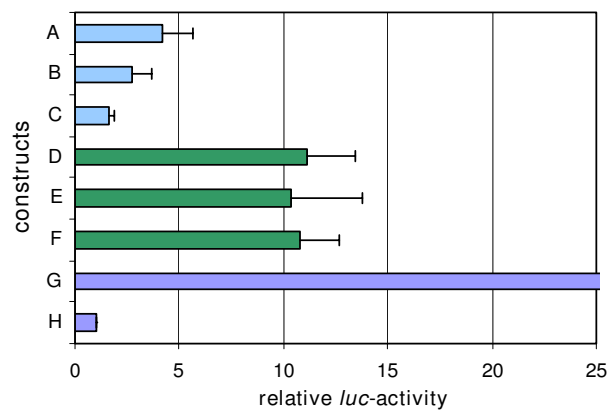

SH-SY5Y

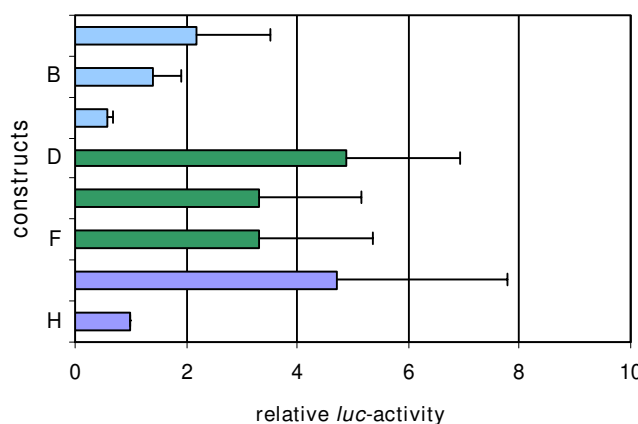

SH-EP

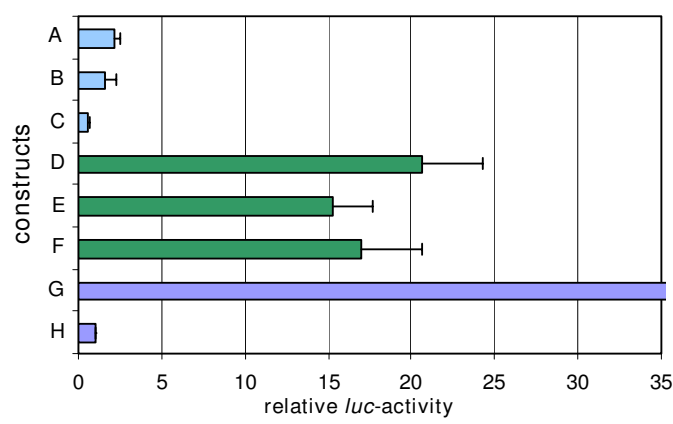

Kelly

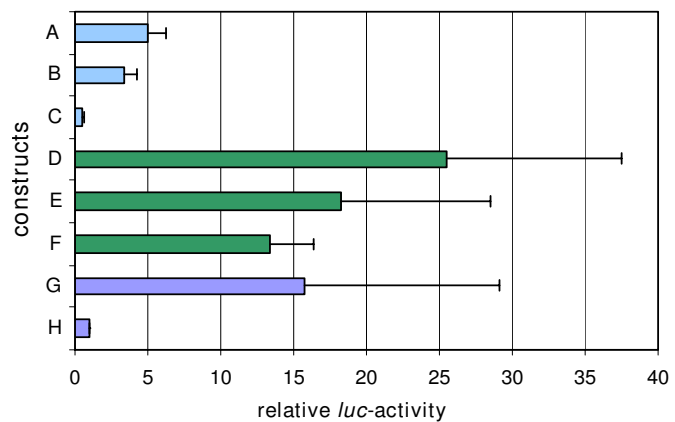

Figure 3

Genomic organization of the human HAND2-DEIN locus on 4q33-34 and luciferase reporter-gene constructs A-H (A); Relative luciferase activity of constructs A-H in NB cell lines SK-N-AS, SH-EP, SH-SY5Y and Kelly

(B). Boxes and lines indicate exons and introns, respectively. Arrows indicate direction of transcription. Blue bars, DEIN orientation; green bars, HAND2 orientation; BP, Bidirectional Promoter; luc, luciferase; BAE, HAND2 Branchial Arch Enhancer; C, putative cis-regulatory element; SVE/SVP, SV40 enhancer- and promoter-elements, respectively. Values of transfection experiments are shown as the mean \pm standard deviation of 3 independent experiments. Values are normalized to the activity of the PGL3-Basic vector set as I. 


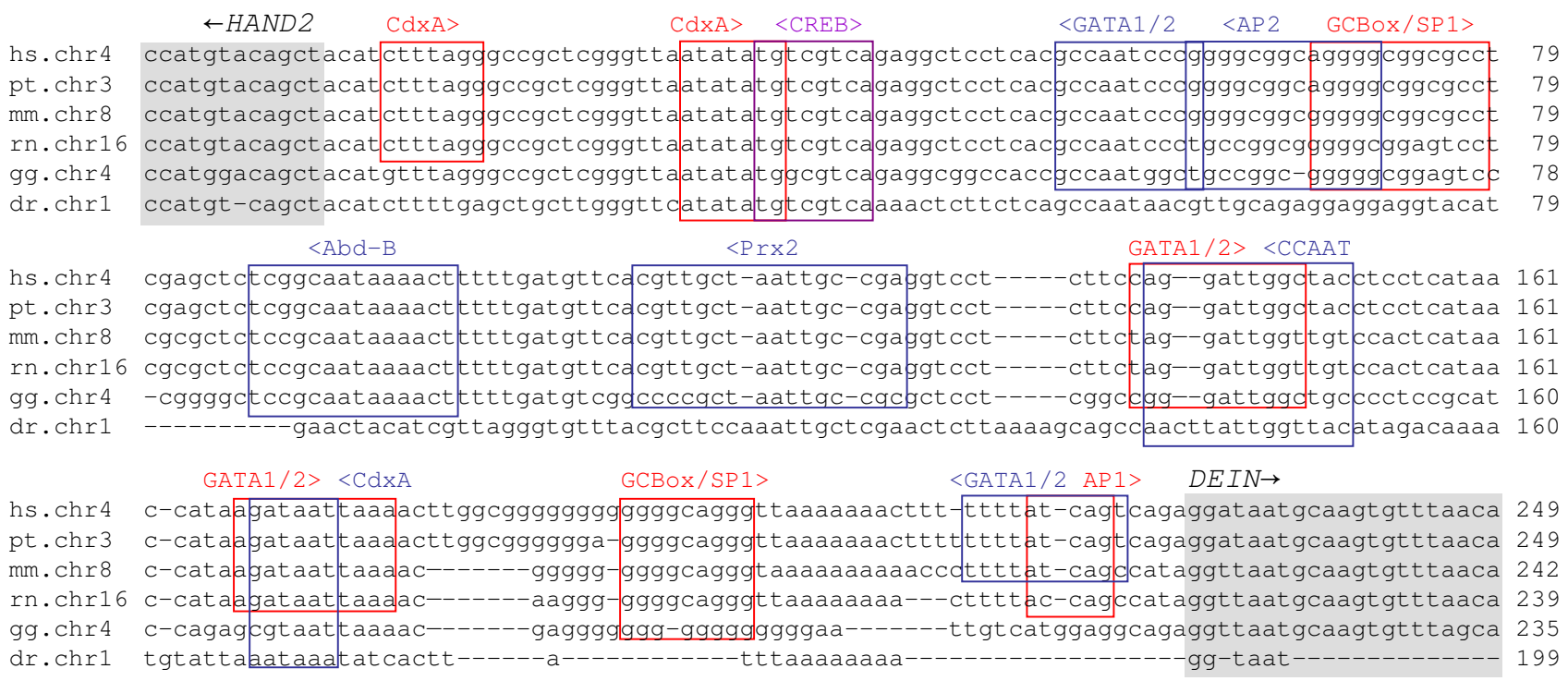

\section{Figure 4}

Sequence alignment of the complete nucleotide sequence of the bidirectional promoter between HAND2 and DEIN in human (hs), chimpanzee (pt), mouse (mm), rat (rn), chicken (gg) and zebrafish (dr). First exons of HAND2 and DEIN are shaded in grey. Direction of transcription is indicated by black arrows. Nucleotides are numbered relative to the first nucleotide of the bidirectional promoter. Putative Trancription Factor Binding Sites (TFBS) are boxed. The directionality of TFBS is indicated by arrowheads and color of boxes (<, blue, HAND2-orientation; $>$, red, DEIN-orientation). SP-I, specificity protein I; AP-I and AP-2, activator protein I and 2; CdxA, CdxA homeobox gene; Abd-B, Abdominal-B; CREB, cAMP response element-binding protein; Prx2, paired related homeobox 2.

The constancy of the intergenic distance between HAND2 and DEIN might thus be indicative of the selective pressure that was acting to maintain the close distance of the genes over a long evolutionary period.

The nucleotide sequence, exon number and transcript structure including intron-exon boundaries of HAND2 are highly conserved among different species including chicken and zebrafish. In contrast, DEIN is only conserved in mouse and chicken but not zebrafish, and shows an overall lower conservation that decreases in 3'-direction with the highest conservation in exons 1 and 2 and a region in exon 5 [6]. Numerous transcribed sequences matching nearly all exons of DEIN were found in mouse and chicken, but not in zebrafish. Tissue sources for these sequences included typical HAND2 expressing tissues such as heart, and embryonic or fetal tissues such as trophoblast and developing sympathetic ganglia, respectively [6]. We conclude from these findings that (1) DEIN has probably first evolved after zebrafish in higher vertebrates, and that (2) bidirectional transcription of HAND2 and DEIN is probably evolutionary conserved in higher vertebrates such as rodents and birds. The latter conclusion is further supported by large-scale comparative analyses of bidirectional promoters in vertebrates using orthology assignments, according to which many bidirectional promoters have emerged after the divergence of birds and fish [18].

\section{Asymmetrical activity of the bidirectional promoter in NB cell lines}

The activity of the bidirectional promoter in four NB cell lines was shown to be asymmetric with strong activity in HAND2 and moderate activity in DEIN orientation. Asymmetric activity has been reported previously for other divergently transcribed gene pairs $[13,19]$.

The comparatively low activity of the promoter in DEIN orientation is surprising regarding the strong expression of DEIN in primary NB as indicated by SAGE, Northern Blot and RT-PCR analysis (fig. 1 and [6]). DEIN expression might possibly require additional cis-regulatory elements that were not included in our constructs. Alternatively, activation of the promoter in DEIN orientation could also depend on transcriptional activators absent in NB cell lines, which are derived from rapidly progressing tumors characterized by rather low DEIN mRNA levels [6]. Transcript levels of both genes might also be further modified by post-transcriptional mechanisms such as mRNA modification or degradation.

Two other highly conserved sequence elements, one of which was shown to function as an enhancer for HAND2 transcription in branchial arches [9], appear to act as weak repressors for both DEIN and HAND2 transcription in NB. However, the in vitro repressive activity could also be 
a result of steric inhibition of transcription factors, since the genetic distance between the two regulatory elements and the promoter in the genome is about $6-8 \mathrm{~kb}$, whereas it is only $44 \mathrm{bp}-1.9 \mathrm{~kb}$ in the luciferase reporter constructs.

\section{Transcriptional regulation and putative functional link of HAND2 and DEIN in NB}

Tissue specific regulation of HAND2 expression by cis-regulatory elements located in its upstream genomic region has been extensively studied in heart and branchial arches, but to date, genomic elements that are critical for HAND2 expression in the developing sympathetic nervous system have not been identified. In heart, HAND2 transcription depends on an enhancer element consisting of two conserved GATA sites that are dispersed over a genomic region of $1.5 \mathrm{~kb}$ between exon 3 and 4 of DEIN with an overall low conservation [20]. In branchial arches, HAND2 expression depends on endothelin-signaling via Dlx6 which binds to a highly conserved 200 bp long enhancer element located between exon 3 and 4 of DEIN [9]. According to McFadden et al. who studied regulatory elements for HAND2 expression in mouse, the genomic region $11 \mathrm{~kb}$ upstream of hand 2 did not direct expression in sympathetic ganglia [20]. This result seems to be in contrast with our finding of a strong transcriptional activation of a reporter gene by the 228 bp upstream region of HAND2 in human NB cells that are suggested to derive from sympathicoadrenal precursors.

Our data indicate that the bidirectional promoter is an important element for HAND2 expression in sympathicoadrenal tissues, although the exact transcriptional activators have not been specified yet. It has been demonstrated earlier that Phox $2 b$ knockout mice lack Hand2 expression [21]. These data strongly suggest that $P H O X 2 B$, a master gene required for the development and differentiation of autonomic neurons [22], is an upstream activator of HAND2 in the developing sympathetic nervous system $[2,3,23]$. Expression analyses of $P H O X 2 B$, HAND2 and DEIN in NB cells lines SH-SY5Y and SH-EP, two well-studied subclones of SK-N-SH with a neuronallike and epithelial-like phenotype, respectively [24], further suggest that not only HAND2, but also DEIN is a target gene of PHOX2B: In cell line SH-EP, PHOX2B expression was shown to be absent [25], and likewise, neither HAND2 nor DEIN was expressed in SH-EP cells according to our Northern Blot analysis (fig. 1); in contrast, all three genes are expressed in cell line SH-SY5Y ([25] and fig. 1). In order to investigate whether DEIN and HAND2 are possible downstream targets of $P H O X 2 B$ in primary $\mathrm{NB}$, we simultaneously examined DEIN and HAND2 mRNA levels in sixty selected tumors with high and low PHOX2B expression values using microarrays (fig. 5). Tumors of both subgroups showed largely con- cordant expression levels of DEIN and HAND2. However, whereas low expression levels of $P H O X 2 B$ were consistently associated with low mRNA levels of DEIN and HAND2 in the vast majority of cases, the degree of correlation appeared to be lower for tumors with high $P H O X 2 B$ expression levels. These observations provide further evidence that $P H O X 2 B$ may act as an upstream activator of both DEIN and HAND2, although additional factors may be necessary to induce expression of these genes in NB. Taking the presence of multiple conserved binding sites for homeobox transcription factors within the bidirectional promoter and the concurrent expression of HAND2 and DEIN in NB into account, it is tempting to speculate that $P H O X 2 B$ could actually represent a direct transcriptional activator for HAND2 and DEIN via the common promoter sequence.

The known PHOX2B downstream targets HAND2 and PHOX2A were shown to promote differentiation of sympathicoadrenal cells and expression of noradrenergic marker genes. In NB cell lines, forced over-expression of wild-type $P H O X 2 B$ was shown to suppress cell proliferation and promote differentiation [26]. As another hypothetical PHOX2B target gene, DEIN might be involved in these cellular processes. This is particularly interesting in the light of the strong DEIN expression in tumors of stage $4 \mathrm{~S}$ and those of children diagnosed $<1$ year of age. Since these tumors are associated with a good prognosis and often follow spontaneous regression, they have been speculated to represent embryonal remnants rather than malignant tumors [27].

Bidirectional gene pairs have been shown to be functionally related, e.g. in DNA repair [7], senescence [11] or brain disease $[13,14]$. The concurrent quantitative and tissue specific expression of both genes ([6], fig. 1 and 2C) suggests that HAND2 and DEIN are regulated by common transcriptional mechanisms in a restricted range of tissues during early developmental stages. The bidirectional promoter probably plays an important role in coordinating HAND2 and DEIN expression in a similar spatial and temporal fashion. However, the functional relevance of the linkage between DEIN and HAND2 expression by the bidirectional promoter requires further investigation, e.g. functional studies on DEIN and experiments elucidating the transcription factors that bind to the promoter region.

\section{Conclusion}

HAND2 and DEIN represent a gene pair that is tightly linked by a bidirectional promoter in an evolutionary highly conserved manner. The activity of the promoter in NB cell lines is asymmetric with strong activity in HAND2 and moderate activity in DEIN orientation. Expression studies strongly suggest co-regulation of both genes, and their high expression in tissues corresponding to the 

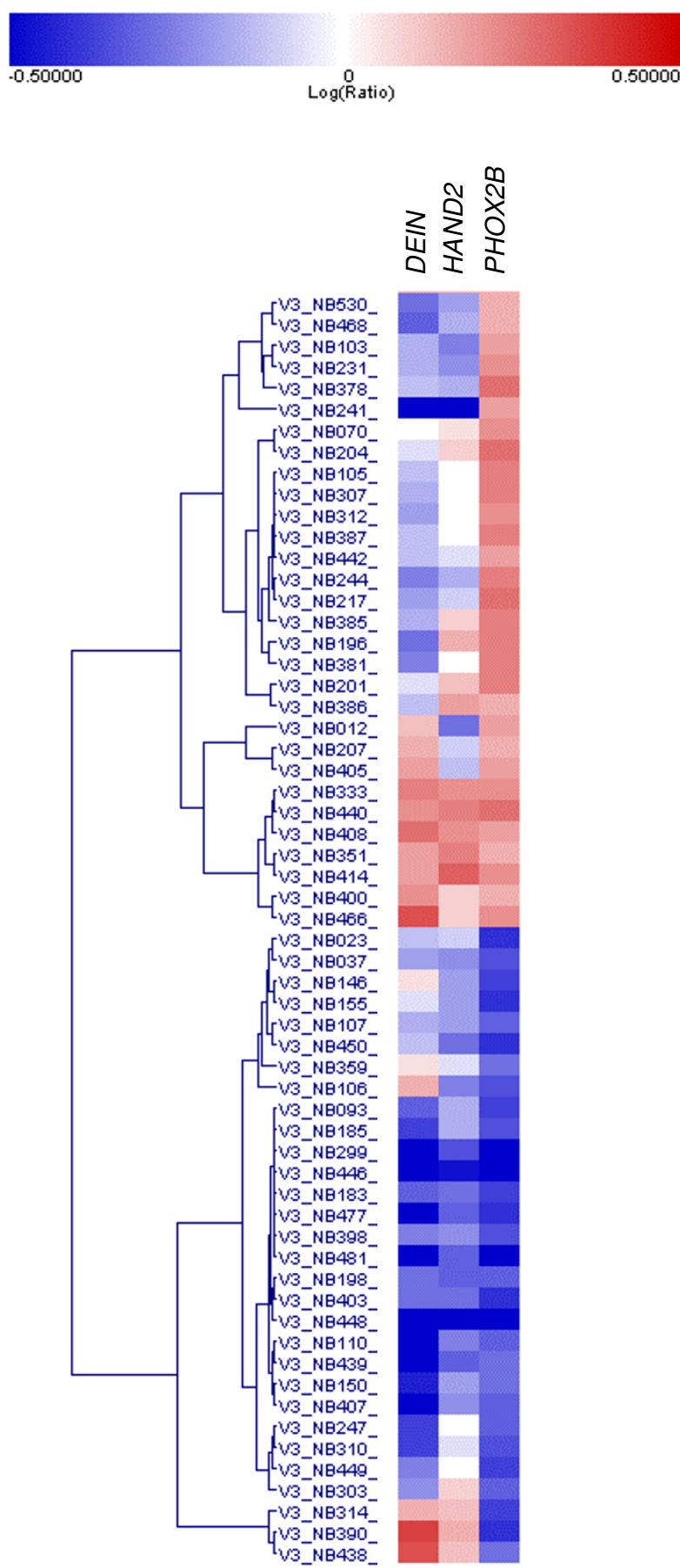

Figure 5

Hierarchical cluster analysis of 60 primary neuroblastomas with high and low PHOX2B expression levels according to expression of DEIN, HAND2 and PHOX2B as determined by microarray analysis. Lines represent patients, columns represent genes. Gene expression levels are visualized as log-values ranging from blue ($0.5)$ to red $(+0.5)$. developing sympathetic nervous system suggests a functional link between HAND2 and DEIN. Co-regulated and tissue specific expression are likely both mediated by the bidirectional promoter, which appears to be evolutionary highly conserved in rodents and birds. Concordant expression levels of HAND2, DEIN and PHOX2B in many primary NB and NB cell lines suggest that $P H O X 2 B$ acts as an upstream transcriptional activator for both genes. Regarding the key role of HAND2 in the differentiation of neural crest derived precursors into sympathetic neurons and the high expression of DEIN in spontaneously regressing NB, DEIN could be instrumental in biological phenomena like regression and differentiation, which are characteristic for favourable NB and the developing sympathetic nervous system.

\section{Methods \\ 3'- and 5'-RACE-PCR}

To identify the 5'-ends of HAND2 and DEIN, 5'-RACEPCR was performed using the SMART ${ }^{\text {тм}}$ RACE CDNA Amplification Kit (Clontech, Mountain View, USA) with the gene specific primers GSPH2 for HAND2 and GSPD1 for DEIN. Cloning of the 3'-end of HAND2 was performed using the SMART ${ }^{\mathrm{TM}}$ RACE cDNA Amplification Kit (Clontech) with the gene specific primer GSPH1 (table 1).

\section{Synthesis of CDNA, cloning and sequencing of PCR products}

Generation of cDNA used for 5'- and 3'-RACE-PCR and RT-PCR was performed with $0.4-2 \mu \mathrm{g}$ of poly(A)-RNA of a primary stage $4 \mathrm{~S}$ neuroblastoma. For RT-PCR, cDNAsynthesis was performed with the QIAGEN LongRange 2Step RTPCR kit according to the manufacturer's protocol. Cloning of PCR products was carried out using the TOPO $\mathrm{TA}^{\oplus}$-Vector (Invitrogen). Sequencing was performed using the BigDye terminator sequencing kit, Version 3.1 (Applied Biosystems).

\section{Reverse transcriptase (RT)-PCR}

PCR was carried out in a total volume of $50 \mu \mathrm{l}$ containing $1 \mu \mathrm{l}$ first strand cDNA, 2 U HiFi Platinum-Taq DNA Polymerase (Invitrogen), $125 \mathrm{nM}$ sense and anti-sense primer each, $20 \mathrm{mM}$ Tris- $\mathrm{HCl}$ (pH 8.4), $50 \mathrm{mM} \mathrm{KCl}, 200$ nM dNTPs each and $1.5 \mathrm{mM} \mathrm{MgSO}_{4}$ (Invitrogen). Cycling conditions consisted of a single denaturation step at $95^{\circ} \mathrm{C}$ for $3 \mathrm{~min}$, followed by 35 cycles of $95^{\circ} \mathrm{C}$ for $1 \mathrm{~min}, 58^{\circ} \mathrm{C}$ for $1 \mathrm{~min}, 72^{\circ} \mathrm{C}$ for $11.5 \mathrm{~min}$ and a final extension step at $72^{\circ} \mathrm{C}$ for $15 \mathrm{~min}$. For the amplification of overlapping subfragments of HAND2, the primer combinations F1 + $\mathrm{R} 4$ and F3 + R3 (table 1) were used. PCR products were visualized on a $2 \%$ agarose gel. As a negative control, RTPCR reactions were performed containing $1 \mu \mathrm{l}$ of first strand cDNA reactions without reverse transcriptase to exclude amplification of contaminating genomic DNA. 
Table I: Oligonucleotides used as primers in this study

\begin{tabular}{|c|c|c|}
\hline method & name & nucleotide sequence \\
\hline 3'-RACE & GSPHI & 5'-CATGGACCTGCTGGCCAAGGACG-3' \\
\hline \multirow[t]{2}{*}{ 5'-RACE } & GSPH2 & 5'-CTGAATGAGCCTTGGAGCTCGAAG-3' \\
\hline & GSPDI & 5'-GCCAATCTGCAGTTTGTCTTTCTG-3' \\
\hline \multirow[t]{10}{*}{ reporter gene constructs } & BPF & 5'-TTTTAAGCTTACATCTTTAGGGCCGCTCG-3' \\
\hline & BPR & 5'-TTTTAAGCTTGTTAAACACTTGCATTATCCTCTG-3' \\
\hline & CDF & 5'-TTTTGGATCCACCTTTCGCCGGAGGCGA-3' \\
\hline & CDR & 5'-TTTTGGATCCAGCGAGCGGCTGCAGATTTG-3' \\
\hline & $\mathrm{CHF}$ & 5'-TTTTACGCGTACCTTTCGCCGGAGGCGA-3' \\
\hline & $\mathrm{CHR}$ & 5'-TTTTACGCGTAGCGAGCGGCTGCAGATTTG-3' \\
\hline & BDF & 5'-TTTTGGGATCCGTTTGTAATAAGAGAATGACCGAA-3' \\
\hline & BDR & 5'-TTTTGGGATCCAGGTCTCCTTGGTAATTTGGGTAC-3' \\
\hline & $\mathrm{BHF}$ & 5'-TTTTACGCGTGTTTGTAATAAGAGAATGACCGAA-3' \\
\hline & BHR & 5'-TTTTACGCGTAGGTCTCCTTGGTAATTTGGGTAC-3' \\
\hline \multirow[t]{13}{*}{ RT-PCR \& Northern Blot } & $\mathrm{FI}$ & 5'-AGCTGTACATGGAGATCTTGC-3' \\
\hline & F2 & 5'-AAAATCAAGACCCTGCGCCTG-3' \\
\hline & F3 & 5'-GCGAAATGAGTCTGGTAGGTG-3' \\
\hline & $\mathrm{F} 4$ & 5'-CGACCCATGTAATATGTAACA-3' \\
\hline & F5 & 5'-AATGGGATTCTCTATTTGTGCTG-3' \\
\hline & F6 & 5'-GAAGGCACAGATCATTCATGG-3' \\
\hline & RI & 5'-CTCACTGTGCTTTTCAAGATTTC-3' \\
\hline & $\mathrm{R} 2$ & 5'-CTTGTCGTTGCTGCTCACT-3' \\
\hline & R3 & 5'-CACAGTGGTTTATTGAATACTTAC-3' \\
\hline & R4 & 5'-CACCT ACCAGACTCA TTTCGC-3' \\
\hline & R5 & 5'-TCAGCTAGAAAACTGTATAAGAG-3' \\
\hline & R6 & 5'-ACCACAAGCAGTCTCATGGGA-3' \\
\hline & R7 & 5'-CAGTAAAAAAAACAGTTTGAAAGGC-3' \\
\hline
\end{tabular}

Forward primers are denoted F, reverse primers are denoted R; GSPHI-GSPDI are gene specific primers for 3'- and 5'-RACE. BPF-BHR were used for amplification of fragments for subsequent cloning of reporter gene constructs $A-F$ (restriction sites are underlined). FI-6 and RI-7 are primers used for RT-PCR and generation of Northern Blot probes.

\section{Northern Blot analysis}

Ten $\mu$ g of total RNA from 20 randomly selected primary NB specimen (stage $1, \mathrm{n}=3$; stage $2, \mathrm{n}=3$; stage $3, \mathrm{n}=3$; stage $4 S, n=5$; stage $4, n=6$ ) and 3 NB cell lines (SH-EP, SH-SY5Y and Kelly) were size-fractioned on a $1 \%$ denaturating formaldehyde agarose gel and transferred onto a nylon membrane (Roche Diagnostics, Mannheim, Germany) using Northern Max One-Hour Transfer Buffer (Ambion, Cambridgeshire, UK). Specific cDNA-probes for DEIN, HAND2 and $\beta$-actin were generated by RT-PCR. Primers used for amplification of specific cDNA-probes were F4 + R5 for DEIN, and F2 + R3 for HAND2 (table 1). Blots were hybridized overnight at $42{ }^{\circ} \mathrm{C}$ in UltraHyb Hybridization Buffer (Ambion) with high sensitivity strippable DNA probes labelled with $\left[\alpha^{32} \mathrm{P}\right]$-dATP (Strip-EZ DNA kit, Ambion). After hybridization, membranes were washed, air-dried and exposed to Kodak BioMax MR-1 films (Amersham, Freiburg, Germany).

\section{Microarray analysis and statistical analysis of expression data} Gene expression profiles from 236 patients (localized stages, $\mathrm{n}=119 ;$ stage $4 S, \mathrm{n}=28$; stage $4, \mathrm{n}=89$; age $<1$ year, $\mathrm{n}=128$; age $>1$ year, $\mathrm{n}=108$ ) were generated as dyeflipped dual-color replicates using customized $11 \mathrm{~K}$ microarrays as described [28]. This set of patients did neither overlap with the cohort of the Northern Blot analysis nor with the cohort of our previous study [28]. Variables of interest (stage and age at diagnosis) and relative DEIN and HAND2 expression levels were compared using Chisquare-test, Kruskal-Wallis test or Mann-Whitney-U-test where appropriate. Correlation of relative expression values was calculated with Pearson's correlation coefficient.

Hierarchical clustering analyses was performed using the Rosetta Resolver Software (Version 7.2; Rosetta Inpharmatics LCC, Seattle). From the whole set of 236 patients, groups with highest $(\mathrm{n}=30)$ and lowest $(\mathrm{n}=30)$ PHOX2B expression were picked and used for the clustering. The analysis was performed using Complete Linkage and Cosine Correlation as metric variables.

\section{Sequence analysis}

The GC-content and CpG islands were determined using CpGplot http://www.ebi.ac.uk/emboss/cpgplot. Homol- 
ogy analysis was performed by comparing sequences of different species with the human sequence of the bidirectional promoter using the ALIGN-webtool, which calculates a global alignment between two sequences http:// www.ch.embnet.org/software/LALIGN form.html. Prediction of putative TFBS was performed using the program Motif http://motif.genome.jp/.

\section{Reporter gene constructs and luciferase assays}

The intergenic region between HAND2 and DEIN was amplified by PCR from human genomic DNA with the primers BPF and BPR (table 1) containing a 10 bp cloning adaptor with a HindIII-restriction site. It was then cloned in both orientations into the HindIII-site of the pGL3Luciferase reporter Vector (constructs A and D, fig. 3; Promega, Darmstadt, Germany).

The 302 bp long putative cis-regulatory element "C" was amplified by PCR with the primers CDF and CDR and cloned downstream of the luciferase gene into the BamHIsite of the DEIN-promoter vector (construct B, fig. 3). For cloning of construct $\mathrm{E}$, " $\mathrm{C}$ " was amplified with the primers $\mathrm{CHF}$ and CHR and cloned upstream of the luciferase gene into the MluI-site of the HAND2-promoter vector (construct E, fig. 3). The 90 bp long enhancer for HAND2expression in branchial arches (BAE) was PCR amplified with the primers BDF and BDR and cloned downstream of the luciferase gene into the BamHI-site of the DEIN-promoter-vector (construct C, fig. 3). For cloning of construct F, "BAE" was amplified with the primers BHF and BHR and cloned upstream of the luciferase gene into the MluIsite of the HAND2-promoter vector (construct F, fig. 3).

All constructs were sequenced with the BigDye Terminator Kit (PE Applied Biosystems, Foster City, USA) to ensure the correct orientation and nucleotide sequence. NB cell lines SH-EP, SK-N-AS, SH-SY5Y and Kelly were cultured in RPMI medium supplemented with $10 \%$ FCS. Cells were transfected using Lipofectamin 2000 (Invitrogen, Karlsruhe, Germany) following the manufacturer's instructions. Approximately $3 \times 10^{4}$ cells were transferred into 96-well plates and transfected with a mixture containing 1 $\mu \mathrm{g}$ of the respective reporter construct and $2 \mathrm{ng}$ of pRLCMV (Promega), which contains the Renilla luciferase gene under the cytomegalovirus SV 40 promoter and enhancer serving as an internal control. Cells were harvested and lysed 27-30 h after transfection and the luciferase activity was detected using the Dual Luciferase Reporter Assay Kit (Promega) and measured with a luminometer (Mitras, Berthold, Germany) according to the manufacturer's instructions. The luciferase activity of the reporter plasmids was normalized to the renilla luciferase acitivity. Each transfection experiment was carried out three times in duplicate and a total of four measurements for each construct were performed. Student's t-test was used to calculate the statistical significance between the relative activities of the various constructs compared to that of the promoterless vector.

\section{Authors' contributions}

$\mathrm{HV}$ is responsible for the conception of the study, the performance of RT-PCR, luciferase reporter assays and sequence analyses, and the writing of the manuscript. AO performed the analysis of microarray expression data. TS carried out the statistical analysis of expression data. YK performed Northern Blots and luciferase reporter assays. FB provided clinical data and contributed to critically revise the manuscript. MF coordinated the study, participated in the design and the writing of the manuscript, and revised it critically for important intellectual content. All authors read and approved the final manuscript.

\section{Additional material}

\section{Additional File 1}

The table indicates expression values (log ratios) of HAND2, DEIN and $\mathrm{PHOX} 2 \mathrm{~B}$ in 236 primary NBs as determined by micoarray analysis, patient ID, stage, MYCN-status (1, single copy; A, amplified) and age at diagnosis (days).

Click here for file

[http://www.biomedcentral.com/content/supplementary/14712199-10-28-S1.xls]

\section{Acknowledgements}

We thank Dr. Roland Ullrich and Gaby Johannsen (Max Planck Institute for Neurological Research, Cologne) for helpful discussions and excellent technical assistance. This work was supported by grants from the Deutsche Krebshilfe (grant 50-2719), the Bundesministerium für Bildung und Forschung (BMBF) through the National Genome Research Network 2 (NGFN2, grant 0IGS0456) and the Competence Network Pediatric Oncology and Hematology (KPOH) as well as the Fördergesellschaft Kinderkrebs-Neuroblastom-Forschung e.V. and the Köln Fortune Program, Faculty of Medicine, University of Cologne (Project 22/2005).

\section{References}

I. Firulli $A B$ : A HANDful of questions: the molecular biology of the heart and neural crest derivatives (HAND)-subclass of basic helix-loop-helix transcription factors. Gene 2003, 3 I 2:27-40.

2. Howard MJ, Stanke M, Schneider $C$, Wu X, Rohrer $H$ : The transcription factor dHAND is a downstream effector of BMPs in sympathetic neuron specification. Development 2000, I 27(18):4073-408I.

3. Hendershot TJ, Liu H, Clouthier DE, Shepherd IT, Coppola E, Studer $M$, Firulli $A B$, Pittman DL, Howard MJ: Conditional deletion of Hand2 reveals critical functions in neurogenesis and cell type-specific gene expression for development of neural crest-derived noradrenergic sympathetic ganglion neurons. Dev Biol 2008, 3 19(2): 179-191.

4. Lucas ME, Muller F, Rudiger R, Henion PD, Rohrer H: The bHLH transcription factor hand 2 is essential for noradrenergic differentiation of sympathetic neurons. Development 2006, 133(20):40I5-4024.

5. Gestblom C, Grynfeld A, Ora I, Ortoft E, Larsson C, Axelson H, Sandstedt B, Cserjesi P, Olson EN, Pahlman S: The basic helix-loophelix transcription factor dHAND, a marker gene for the 
developing human sympathetic nervous system, is expressed in both high- and low-stage neuroblastomas. Lab Invest 1999, 79(I):67-79.

6. Voth H, Oberthuer A, Simon T, Kahlert $Y$, Berthold F, Fischer M: Identification of DEIN, a novel gene with high expression levels in stage IVS neuroblastoma. Mol Cancer Res 2007, 5(I 2): 1276-1284.

7. Adachi N, Lieber MR: Bidirectional gene organization: a common architectural feature of the human genome. Cell 2002, 109(7):807-809.

8. Russell MW, Kemp P, Wang L, Brody LC, Izumo S: Molecular cloning of the human HAND2 gene. Biochim Biophys Acta 1998, I 443(3):393-399.

9. Charite J, McFadden DG, Merlo G, Levi G, Clouthier DE, Yanagisawa $\mathrm{M}$, Richardson JA, Olson EN: Role of Dlx6 in regulation of an endothelin-I-dependent, dHAND branchial arch enhancer. Genes Dev 200I, I 5(22):3039-3049.

10. Trinklein ND, Aldred SF, Hartman SJ, Schroeder DI, Otillar RP, Myers RM: An abundance of bidirectional promoters in the human genome. Genome Res 2004, I4(I):62-66.

11. Bellizzi D, Dato S, Cavalcante P, Covello G, Di Cianni F, Passarino G Rose G, De Benedictis G: Characterization of a bidirectional promoter shared between two human genes related to aging: SIRT3 and PSMDI3. Genomics 2007, 89(I): I43-I50.

12. Wright KL, White LC, Kelly A, Beck S, Trowsdale J, Ting JP: Coordinate regulation of the human TAPI and LMP2 genes from a shared bidirectional promoter. J Exp Med 1995, I 8 I(4): |459-|47|.

13. Chen PY, Chang WS, Chou RH, Lai YK, Lin SC, Chi CY, Wu CW: Two non-homologous brain diseases-related genes, SERPINII and PDCDI0, are tightly linked by an asymmetric bidirectional promoter in an evolutionarily conserved manner. BMC Mol Biol 2007, 8:2.

14. West $A B$, Lockhart PJ, O'Farell C, Farrer MJ: Identification of a novel gene linked to parkin via a bi-directional promoter. J Mol Biol 2003, 326(I): I I-I 9.

15. Greene WK, Sontani Y, Sharp MA, Dunn DS, Kees UR, Bellgard MI: A promoter with bidirectional activity is located between TLXI/HOXII and a divergently transcribed novel human gene. Gene 2007, 39I(I-2):223-232.

16. Xu CF, Brown MA, Nicolai H, Chambers JA, Griffiths BL, Solomon E: Isolation and characterisation of the NBR2 gene which lies head to head with the human BRCAI gene. Hum Mol Genet 1997, 6(7): 1057-1062.

17. Shu J, Jelinek J, Chang H, Shen L, Qin T, Chung W, Oki Y, Issa JP. Silencing of bidirectional promoters by DNA methylation in tumorigenesis. Cancer Res 2006, 66 (10):5077-5084.

18. Yang MQ, Taylor J, Elnitski L: Comparative analyses of bidirectional promoters in vertebrates. BMC Bioinformatics 2008, 9(Suppl 6):S9.

19. Travers MT, Cambot M, Kennedy HT, Lenoir GM, Barber MC, Joulin $V$ : Asymmetric expression of transcripts derived from the shared promoter between the divergently oriented ACACA and TADA2L genes. Genomics 2005, 85(I):7I-84.

20. McFadden DG, Charite J, Richardson JA, Srivastava D, Firulli AB, Olson EN: A GATA-dependent right ventricular enhancer controls dHAND transcription in the developing heart. Development 2000, 127(24):5331-534I.

21. Huber K, Karch N, Ernsberger U, Goridis C, Unsicker K: The role of Phox2B in chromaffin cell development. Dev Biol 2005, 279(2):50I-508.

22. Pattyn A, Morin X, Cremer H, Goridis C, Brunet JF: The homeobox gene Phox $2 b$ is essential for the development of autonomic neural crest derivatives. Nature 1999, 399(6734):366-370.

23. Goridis $\mathrm{C}$, Rohrer $\mathrm{H}$ : Specification of catecholaminergic and serotonergic neurons. Nat Rev Neurosci 2002, 3(7):53I-54I.

24. Ross RA, Spengler BA, Biedler JL: Coordinate morphological and biochemical interconversion of human neuroblastoma cells. J Natl Cancer Inst 1983, 7 I (4):741-747.

25. van Limpt $V$, Schramm A, van Lakeman A, Sluis $P$, Chan A, van Noesel M, Baas F, Caron H, Eggert A, Versteeg R: The Phox 2B homeobox gene is mutated in sporadic neuroblastomas. Oncogene 2004 23(57):9280-9288.

26. Raabe EH, Laudenslager M, Winter C, Wasserman N, Cole K, LaQuaglia M, Maris DJ, Mosse YP, Maris JM: Prevalence and func- tional consequence of PHOX2B mutations in neuroblastoma. Oncogene 2008, 27(4):469-476.

27. Castleberry RP: Neuroblastoma 33(9): I430-| 437. discussion |437-|438

28. Oberthuer A, Berthold F, Warnat P, Hero B, Kahlert $Y$, Spitz R, Ernestus K, Konig R, Haas S, Eils R, et al.: Customized oligonucleotide microarray gene expression-based classification of neuroblastoma patients outperforms current clinical risk stratification. J Clin Oncol 2006, 24(3 I):5070-5078.
Publish with Biomed Central and every scientist can read your work free of charge

"BioMed Central will be the most significant development for disseminating the results of biomedical research in our lifetime. "

Sir Paul Nurse, Cancer Research UK

Your research papers will be:

- available free of charge to the entire biomedical community

- peer reviewed and published immediately upon acceptance

- cited in PubMed and archived on PubMed Central

- yours - you keep the copyright 